\title{
Anti-Unfair Competition Law (Revised Draft) - Studies on the Reasonableness of Grant Supervision and Inspection Authorities to Seizure and Detention Right
}

\author{
Yue Zhang ${ }^{1, a}$, Siming Zhang ${ }^{2, b}$, Quanyi Li ${ }^{3, c}$ \\ ${ }^{1}$ Capital University of Economics and Business, Beijing,China \\ ${ }^{2}$ Capital University of Economics and Business, Beijing,China \\ ${ }^{3}$ Capital University of Economics and Business, Beijing,China \\ a769497917@qq.com,b1961009805@qq.com,c1335083020@qq.com
}

Key world: Anti-Unfair Competition Law, Revised draft, Supervision and inspection organs, Seizure of seizure

\begin{abstract}
Since 1993, the Anti-Unfair Competition Law of the People's Republic of China (hereinafter referred to as the Anti-Unfair Competition Law) has been applied since its inception. However, with the rapid development of economy, economic model, industrial pattern, the size of the market changes, in order to improve China's market supervision system to combat competition in various areas of behavior, China in 2016 proposed "anti-unfair competition law draft)". Article 16 of the new Article 16 of the "supervision and inspection departments in the investigation of unfair competition, the right to exercise seizure, seizure and suspected of unfair competition behavior related to the property". According to the law, the supervisory organ of unfair competition is the business administration department above the county level. The addition of the clause is undoubtedly the expansion of the powers of the business administration. Will the granting of coercive measures affect the abuse of power? Will there be a greater threat to the rights of the executive counterparts? Can the legal quality of law enforcement officers be able to implement the power correctly? Whether it is possible to achieve the desired purpose of the mandate ... This series of questions is what we should be concerned about.
\end{abstract}

\section{《反不正当竞争法（修订草案）》 授予监督检查机关查封扣押权的合理性研究 \\ 张悦 $1, \mathrm{a}$, 张思名 $2, \mathrm{~b}$, 李泉怡 $3, \mathrm{c}$ \\ 1 首都经济贸易大学, 北京, 中国 \\ 2首都经济贸易大学, 北京, 中国 \\ 3 首都经济贸易大学，北京，中国 \\ a769497917@qq.com,,1961009805@qq.com,c1335083020@qq.com}

关键词: 反不正当竞争法 修订草案 监督检查机关 查封扣押权

摘要:自 1993 年《中华人民共和国反不正当竞争法》（以下简称《反不正当竞争法》）开始 施行, 一直适用至今。但随着经济的飞速发展, 经济模式、产业格局、市场规模的随之变化, 为了完善我国的市场监管制度，打击各领域限制竞争的行为，我国于 2016 年提出《反不正当 竞争法（修订草案）》。其中第三章第十六条新增“监督检查部门在调查不正当竞争行为时, 有权行使查封、扣押与涉嫌不正当竞争行为有关的财物”的职权。根据法律规定，不正当竞争 
行为的监督机关是县级以上的工商管理部门。该项条款的增设无疑是扩大了工商管理部门的 职权。对于该项强制措施职权的授予是否会导致权力的滥用? 是否会对行政相对人的权利造 成更大的威胁? 执法人员的法律素质是否能够正确的实施该权力? 是否可以实现该项职权预 期的目的......这一系列的问题都是我们应该予以关注的。

\section{1. 查封、扣押等财产性强制措施的法律属性界定}

《行政强制法》从 1999 年起草, 直至 2011 年 6 月在全国人大常务委员会第二十一次会 议通过，2012 年 1 月 1 日起实施。其出台经历了非常艰难的过程，与《行政处罚法》和《行 政许可法》共同形成了我国行政程序立法的初步体系。《行政强制法》主要包括行政强制措 施和行政强制执行两部分, 其作为具体行政行为的一种较为特殊的种类, 其实施将直接影响 公民的基本权利，从其起草到出台所经历的关于“公民权”和“行政权”权衡上的数次博弯，不 难看出, 一直以来对行政强制权的规范和控制都是立法主体和人们所关注的重点。其中关于 该职权的设定主体、实施主体、种类、实施程序、执行程序、法律责任等相关内容规定都极 为严格。查封、扣押作为行政强制措施的主要类型之一, 我国对行政强制权的严格规定即是 对查封、扣押等财产性强制措施的严格规定。以下将根据现行法律法规关于行政强制权（其 中主要依据查封、扣押等财产性强制措施的相关规定）的各项规定来阐明对其严格控制的必 要性。

\section{2. 严格控制查封、扣押权的必要性}

\section{1 行政强制权的设定主体}

行政强制的原则之一——行政强制法定原则，其主要包含行政强制设定法定和行政强制 实施法定两方面含义。行政强制的设定权一般只能是法律, 行政法规、地方性法规等是否具 有设定权取决于法律是否对其进行授权。这也是《行政强制法》第四条的规定。

详细来说，行政强制执行的设定更为严格，只能有法律进行创设。而行政强制措施的设 定权则相比而言较为宽松。法律可以设定所有的行政强制措施，但却保留了限制人身自由和 冻结存款、汇款的行政强制措施。因此，行政法规可以就尚未制定法律的事项以及法律授权 可以设定的行政强制措施进行创设。就地方性法规而言, 可以设定查封场所、设施、财物和 扣押财物有关的行政强制措施。而规章、文件等均无设定行政强制的权利。法律未设定的行 政强制措施, 行政法规、地方性法规均不得自行创设; 除此之外, 法律对行政强制措施的各 项规定，行政法规、地方性法规也均不得做出扩大规定。1

对行政强制措施设定权的严格规定，足以看出该权力从设定阶段就极为谨慎，法律直接 做出详细、明确的规定，上至行政法规，下至地方性法规均无自行创设甚至扩大规定的权力。 查封、扣押等财产性强制措施作为行政强制措施的一部分，其设定权也受相应的约束。

\section{2 行政强制权的实施主体}

行政强制法定原则之行政强制实施法定要求：行政强制措施只能由行政机关和法律、法 规授权的组织予以实施, 其他任何机关和个人均无权实施。同时这也是《行政强制法》第二 十二条规定。且一律得不委托其他行政机关或者组织实施。其中，查封、扣押权的实施主体 主要有法律法规规定的行政机关和法律、行政法规授权的具有管理公共事务职能的组织。除 了对实施机关的明确规定，同时对查封、扣押的行政执法人员要求具备一定的资格。不仅如 此，对实施查封、扣押还有人员数量的要求，要求至少两名行政执法人员共同实施。

从对有权实施该职权的机关的限制到执法主体资质和人数的严格要求。由此可见，行政 强制措施权力的下放不仅谨慎，同时还尽可能明确的作出相关规定，以防止该职权被未经法

1 王冰: 《地方性法规中行政强制措施的设定权研究》, 华东政法大学, 2014 年 
律授权的组织或个人越权滥用或者已经授权的组织未按规定肆意的行使。除此之外, 行政强 制措施不得委托的规定也体现了对行使该职权人员专业性的要求和对职权的规范、控制。

\section{3 行政强制权的实施限制}

行政强制原则之行政强制适当原则，明确指明行政强制的设定和实施，应当尽可能实现 手段和目的的关系比例适当，能够采取非强制手段可以达到行政管理目的的，即不得设定行 政强制措施。这也是《行政强制法》第五条明文规定。足以看出对行政强制权的设定和实施 都有极高的要求，不可轻易采取行政强制手段。

但在现实中，往往出现“无论如何，先扣下来再说”的现象。这使得该权力不仅未合法、 合理的使用, 同时也损害了相对人的利益。《中华人民共和国宪法》第十三条明确规定, 公 民的合法私有财产不受侵犯。行政机关使用该职权的任意性，侵犯了宪法所保护的人们的私 有财产的权利。若相对人采取法律救济程序，国家因此对执法主体的违法执法行为给相对人 造成的损失做出赔偿, 最终滥用的强制措施损害的却是国家的利益。这种现象不难看出, 2 查 封、扣押等强制措施已经被作为行政机关执法人员行使职权进行监管的一种常用的手段。然 而, 从行政强制的适当原则来看, 查封、扣押作为一种强制措施, 并不应该被作为一种常用 的手段，而应是在束手无策的时候的一种应急手段。

\section{3. 反不正当竞争的监督检查部门在行使查封、扣押职权中可能存在的问题}

\section{1 监督检查部门权力的滥用}

根据法律规定，不正当竞争行为的监督机关是县级以上的工商管理部门。随着法律对其 赋予的行政权能的增加, 意味着, 在不正当竞争的案件中, 其自由裁量的权力和范围也会随 之扩大。执法主体能否公平的对待所有的经营主体？或者在日常的监督调查中能否公正的处 理案件? 都是值得深思的问题。若执法主体主观的目的和动机有所不良, 在对经营者的竞争 行为进行正当性审查的时候采取区别对待则严重违反了公平、公正的要求。因工商管理部门 职能的多样性, 其不仅仅有对不正当竞争行为进行监督检查的职能, 还具有确认市场主体资 格、商标的注册和管理等一系列的职能。对其他事物的处理或者程序上与相对人发生的纠纷 是否会影响其在进行不正当竞争行为检查监督时的公正性？是否会因此而滥用查封、扣押的 权力?

\section{2 对查封、扣押对象的正确性把握难度大}

2014 年 1 月本溪市政协委员、鞍山市人大代表袁诚家因组织、领导黑社会性质组织罪等 多项罪名被判处有期徒刑 20 年, 法院还追缴、没收其财物, 包括 20 多家企业、大量动产与 不动产。2015 年 11 月又将大部分无证据证明为非法财产的部分予以返还。2017 年 5 月袁向 辽宁省公安厅申请国家赔偿 37.3 亿元。其中包含为返还的合法财产、查封、扣押期间本应所 得的营业收益等。国家赔偿法规明文规定, 违法的查封、扣押、冻结应该予以赔偿。对袁合 法财产的扣押无疑是违法的, 所以对其进行赔偿似乎也无可厚非。但这不是我们所讨论的重 点。重点是从这个案例不难看出查封对象的错误, 最终可能给相对人造成的损失, 终究需要 国家来“买单”。工商机关的执法人员相对而言法律素养有所欠缺, 也并非系统全面的去深度 挖掘法条。将查封、扣押职权赋予工商管理机关, 其是否可以正确的把握查封、扣押对象? 是否能明确的区分哪些为违法结果的资产? 哪些物品为违法行为的工具? 哪些是不得查封、 扣押的对象? 现实中, 工商机关的执行人员精通工商管理方面的业务知识, 但是对法律法条 的认识, 却相对模糊。

\footnotetext{
2 《查封、扣押,是常态还是应急?——关于实施查封、扣押的十六个重点问题》，《环境经济》，2015 年 01 期
} 


\section{3 查封、扣押权与行政处罚相混淆}

行政处罚是行政机关对相对人的违法但未犯罪的行为给予行政制裁的具体行政行为。本 文所论述的主要是关于财产性的行政处罚。我国立法对行政处罚的具体适用、程序等都做了 较为详细的规定, 行政处罚一经做出即是对相对人财产所有权的最终处分。而查封、扣押是 对相对人财产的一种暂时性的限制。因此, 现实中, “无论如何, 扣下再说”即先查封、扣押 再决定是否符合行政处罚的情形也不是不可能发生。

2007 年湘潭市公安局岳塘分局就湘潭耀舜环保工贸有限公司涉嫌侵犯商业秘密案立案侦 查。该分局以办案需要为由扣押耀舜公司的财产，并对部分财产进行拍卖。2009 年 3 月 10 日湘潭市岳塘区人民检察院以潭岳检刑诉[2009]41 号起诉书指控赔偿请求人湘潭耀舜环保工 贸有限公司法定代表人张雨新、股东段继平犯侵犯商业秘密罪。同年八月法院作出判决书宣 告张雨新、段继平无罪。随后检察院提起抗诉, 但最终法院裁定驳回抗诉, 维持原判。该分 局就已拍卖部分退还拍卖所得的价款 100800 元，未拍卖部分予以返还，其他损失不予赔偿。 2010 年 11 月 16 日, 湘潭耀舜环保工贸有限公司向赔偿义务机关湘潭市公安局岳塘分局申请 国家赔偿, 经过双方数次博弯, 最终该分局支付国家赔偿款项 227078 元, 其余请求被驳回。 3

该案例则典型的反映了现实中“无论如何，先扣下来再说”的现象。该公安分局对该企业 采取的扣押无疑是“盲目”、“冲动”的，其中对于非法拍卖的指控，也是因为无证据而不成立， 但这不能代表没有非法拍卖的可能性。最终法院判处该企业无罪, 因扣押拍卖所侵犯的该企 业的财产最终虽然得到部分赔偿，但该赔偿远远不及企业所损失的部分。归根结底，因为该 机关查封权力的滥用，不仅仅侵犯了公民的合法财产，同时也损害了国家的利益。

反不正当竞争的监督机关所面临的是纷繁复杂的市场现状和数以万计的市场主体，面对 层出不穷的各类破坏市场秩序的案件, 若其在行使查封、扣押权也“无论如何, 先扣下来再说”, 那么最终要解决的民与官的纠纷可能就不计其数了。

\section{4 腐败的滋生}

经营者从创办到运营企业, 与工商管理机关的联系最为紧密, 注册登记找工商, 运营监 督也是工商。包括每年一次的年检, 也是到工商机关完成。甚至连广告发布、地方性广告核 准手续也是在工商。这就意味着整个企业的“诞生一一成长一一生存”各个环节都与工商紧密 的联系起来, 想要自己的企业发展壮大, 不仅需要自身妥善的经营, 同时也需要顺利完成法 定需要的各个阶段的手续和核准。且《中华人民共和国反不正当竞争法》中详细规定了法人、 其他经济组织和个人的不正当竞争的若干情形。其中不乏监督检查机关即工商机关可自由裁 量的情形。企业的命运虽不至于受其决定, 但或多或少会受其影响。为什么人们会对行政机 关产生畏惧, 不仅仅是因为它代表着党和国家在行使自己的职权, 同时也因为它的手里牢牢 拽着我们的权利。就企业而言, 拥有更多职权甚至强制权利的工商机关自然会是经营者产生 畏惧，因此不得不逢迎讨好，腐败就会在这样不平等的关系中孕育而生。

\section{5 监督检查机关独立性较弱}

对于查封、扣押这样的强制措施必须要求作出决策的主体具有较强的独立性，因为这样 的强制措施一经做出, 必然会给相对人造成一定的财物或者声誉上的损失。所以对这类措施 的决定必然要求谨慎而为之, 要求决定主体尽可能的不受多余因素的影响, 仅就事实本身做 出裁量。但是监督检查机关即工商管理机关是层级结构分明的机关。下级对上级的决定时服 从，除此之外，现实中，不乏下级处事须看上级眼色的现象存在。若对上级特别“关照”的企

\footnotetext{
3 湖南省湘潭市中级人民法院: 《湘潭耀舜环保工贸有限公司请求国家赔偿案》, 法治政府网, 2011 年 11 月 26 日
} 
业, 实际的监督检查人员务必会放松监管力度。而对无权无势的企业, 也不能排除不故意刀 难的可能。当这样的非难成为一种日常，对企业的影响也是可以估量的。

\section{6 相对人的救济问题}

我国现目前的《行政诉讼法》中关于违法的行政行为的救济办法主要是申请行政复议和 提起诉讼。详细来说, 即行政相对人对自己权利受到侵害的行政行为可以申请行政复议, 对 复议不服, 可以再提起行政诉公; 当然也可以直接提起诉公。但是现实生活中, 对于涉及利 益不大的案件, 相对人并不会选择与行政机关做对, 而选择隐忍。当然这是行政相对人自己 选择的结果。但是如果授予工商机关查封、扣押的权力, 若权力遭到滥用, 从本质上来看, 最终还是侵犯了相对人的权利，而法律存在的目的不应该是惩处与保障吗?

另外，在现实中，当查封、扣押作为一种常用的手段时，行政机关做出查封、扣押决定 的时候往往不会告知相对人可以申请救济的时限。最终即便返还了被查封、扣押的财物, 相 对人也没有办法获取理所应当的补偿。所以，授予工商管理机关在调查不正当竞争行为时， 有权行使查封、扣押与涉嫌不正当竞争行为有关的财物的权力, 直接给相对人带来的财物损 失的救济也是值得考虑的问题之一。

\section{References}

[1] Bing Wang. Studies on the Establishment Right of Administrative Coercive Measures in Local Laws and Regulations[D]. East China University of Political Science and Law, 2014.

[2] Zhuohui Zhou. On Civil Execution Seizures[D]. China University of Political Science and Law, 2006.

[3] Shan Wu. Studies on Compulsory Measures System Improvement of China's Criminal InvolvedProperty[D]. Southwestern University of Finance and Economics, 2012.

[4] Zhonggen Zhao. On Seizure and Detention in Administrative Coercive Measures[D]. Southwest University of Political Science and Law, 2014.

[5] Is Seizure, Detention Normal or Emergency? - Sixteen Key Issues on the Implementation of Seizure, Detention[J]. Environmental Economy, 2015,(01):6.

[6] Songlin Wang. Studies on Seized and Detained Property Disposal in Administrative Law Enforcement[A]. Studies on Government Legal System in 2009[C].:, 2009:44.

[7] Yanfang Jia, Lei Shi. Existing Problems and their Causes of Administrative Seizure and Detention in Law Enforcement[J]. Governmental Legal System, 2009, (01):46-47.

[8] Zhongle Zhan. Seizure and Detention in Administrative Coercion[N]. China Industrial and Commercial Daily, 2012-04-12 (A03). 